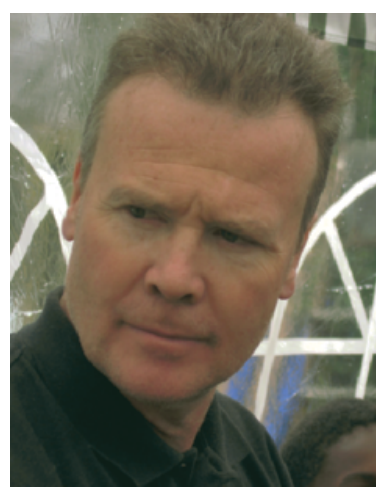

G. Dine

\section{Accréditation et innovation}

Au sein des professions de santé françaises les biologistes ont été les premiers à mettre en place l'évaluation de leur pratique, et ce, bien avant que cette démarche ne soit rendue obligatoire. La mise en place du contrôle national de qualité est à l'initiative de la profession. Son organisation réglementaire par les agences de santé, ensuite, est devenue une réalité professionnelle qu'on pourrait qualifier aujourd'hui de culturelle. II y a une dizaine d'années, le GBEA était présenté comme une évolution majeure visant à assurer la qualité des prestations analytiques de la biologie clinique française. II s'agissait d'une démarche plus institutionnelle et plus directive qui, en son temps, a déclenché confrontations et polémiques. Dans le secteur privé, certains collègues n'ont pas hésité à se lancer dans la voie de l'accréditation au sens industriel du terme de façon volontaire. Cette expérience, si on peut dire, a été souvent regardée comme exotique par beaucoup d'entre nous, exerçant en secteur public. A côté du contrôle national de qualité devenu réglementaire, il faut rappeler l'existence d'innombrables contrôles de qualité volontaires et spécialisés, émanant de sociétés savantes, de groupes scientifiques et de partenaires industriels. En clair, la biologie clinique française est à la pointe de l'évaluation de ses prestations par rapport aux autres spécialités médicales. Dans le cadre de la loi HPST, la place réservée à l'accréditation des laboratoires de biologie clinique est conséquente. Son caractère obligatoire suscite l'interrogation et certaines oppositions. La désignation du COFRAC, comme interlocuteur unique, est en soi un objet de questionnement vis-à-vis des tâches à accomplir pour assurer les étapes 2013-2016 qui ont été affichées. Le fait que seuls les laboratoires accrédités pourront exercer apparait pour beaucoup comme un couperet visant à restreindre le nombre de laboratoires de biologie clinique en France. Le rationnel annoncé pour cette initiative réglementaire s'appuie sur la nécessité de défendre la profession biologique et la spécificité française au plan européen. La qualité du rendu des résultats doit devenir la culture de base. Pour parcourir ce chemin, les regroupements vont être nécessaires pour justifier l'investissement lié au coût de l'accréditation obligatoire. Le nombre de laboratoires d'analyse biologique va donc diminuer dans le pays. Cette opération comptable non déclarée n'a pas échappé aux responsables élus de la profession. Selon les projections, on peut penser qu'à l'horizon 2020 il ne restera qu'un millier de laboratoires privés et que le nombre de laboratoires du secteur public sera compris entre 200 et 300. La notion de proximité peut être mise à mal. Le risque de paupérisation ne peut pas être exclu. L'impact sur la démographie professionnelle est une autre facette qui doit être prise en compte ainsi que les emplois de techniciens. Vis-à-vis de la loi HPST et son versant sur la biologie clinique, on peut être interrogatif quant à la place de l'innovation technologique. Si l'automatisation a entrainé depuis 20 ans un mouvement centripète avec concentration des analyses sur des structures de grande taille, il est vraisemblable que la mise à disponibilité de systèmes «SMART » faisant appel notamment aux nanotechnologies, risque de modifier un peu cette tendance. Certaines analyses très pointues, nécessaires par exemple à l'utilisation des thérapies ciblées vont devenir accessibles à l'emploi de terrain, pour ne pas dire dans le bureau du clinicien, échappant ainsi à la centralisation sur quelques plateformes qui risquent d'apparaître rapidement comme des mastodontes inutiles et coûteux. Ce sont tous les concepts de médecine personnalisée, actuellement en plein développements technologiques, industriels et professionnels qui doivent être appréhendés. En parallèle des JIB 2010 vont se dérouler dans notre pays de nombreuses réunions nationales et internationales sur le sujet. Cette tendance centrifuge est soutenue par les sciences de l'ingénieur et la nécessité économique face aux coûts des thérapeutiques nouvelles. II serait dommageable que la réalité administrative à venir de l'exercice de la biologie clinique placée sous le contrôle de la loi HPST ne soit pas en adéquation avec les modifications de la pratique professionnelle induites par l'innovation technologique. II ne faudrait pas en effet nous réveiller en 2020 avec l'impression désagréable d'avoir été en retard d'un combat. • 